${ }^{1}$ CONACYT-Universidad Autónoma de Coahuila, México. Correo electrónico:

ignaciocruz@apolo.acatlan.unam.mx

\section{DESIGUALDA NUMÉRICA} ENTRE HOMBRE Y MUJERES EN LOS CONSEJOS DE ADMINISTRACIÓN EN GRANDES EMPRESAS MEXICANA IGNACIO JAVIER CRUZ1

\title{
Resumen
}

El objetivo de esta investigación es mostrar la desigualdad numérica existente en los consejos de administración de grandes empresas mexicanas que favorece al género masculino. El ascenso laboral femenino es focalizado como instrumento para alcanzar un puesto en dichos consejos. Se lleva a cabo un conteo del número de mujeres miembros de consejos de administración dividido por tipo de consejeros. Los resultados indican que las mujeres ocupan entre 3.8 y $8 \%$ de los lugares existentes en consejos de administración, cifras que están muy por debajo de la evidencia internacional.

Palabras Clave: desigualdad de género, consejo de administración, empresa, ascenso laboral, México.

\section{Abstract}

The objective of the research is to show the numerical inequality in management board in Mexican enterprises. The job promotion 
is considered to be the way to reach a spot in management broad. I account the number of women members of management boards divided by type. The results indicate that only between 3,8 and $8 \%$ percent of women have a position in management boards this amount is below the international evidence.

Keywords: Gender Inequality, Management boards, enterprise, job promotion, Mexico.

RECEPCIÓN: 28 DE MARZO DE 2017/ACEPTACIÓN: 12 DE JUNIO DE 2017

\section{INTRODUCCIÓN}

Las desigualdades de género en México han estado presentes por muchos años en diversos ámbitos de la vida nacional, desde aspectos religiosos (Villalobos, 2016), hasta educativos (Navarro, Narro y Orozco, 2014). En esta investigación se pretende profundizar en la parte del acontecer empresarial, en particular, en los consejos de administración de grandes empresas con el objetivo de explorar las diferencias numéricas de género en la composición de dichas figuras administrativas.

Llegar a formar parte de un consejo de administración puede ser una tarea difícil por lo que este hecho se enfocará como ascenso laboral. Es decir, ocupar un lugar en un consejo de 
administración de una empresa de gran tamaño puede ser el resultado de haber escalado la estructura administrativa de una o varias organizaciones. Es la consecuencia de haber ascendido. Bajo condiciones de igualdad de género, esta situa-

${ }^{2}$ No debe perderse de vista que la existencia de mujeres en los puestos del consejo se puede dar también por la simple razón que pertenecen a la familia que lo controla. ción se reflejaría en que una buena parte de los puestos de los consejos estarían ocupados por mujeres, pero se mostrará que no es así, ${ }^{2}$ pues la presencia de mujeres en tales consejos es muy escasa. Es necesario mencionar que tanto la inequidad numérica de los consejos como la discriminación en los ascensos laborales son cosas diferentes, pero a lo largo de la investigación se mostrará su relación.

Existe una literatura cuantiosa que versa sobre el impacto de la existencia de inequidad de género en el ascenso laboral de las mujeres. Particularmente, se señala que tal inequidad representa un obstáculo, en términos personales para las mujeres y en términos generales para el desempeño de las empresas y el crecimiento económico de una nación. Este fenómeno es también conocido como "techo de cristal", que según Hernández (2004: 447). Al decir de la autora es un conjunto de normas no escritas que están presentes en la cultura de la empresa y que impiden el acceso de las mujeres a la cúspide.

Para cumplir con el objetivo de mostrar la inequidad numérica de género en la composición de consejos de administración este artículo se divide en tres partes. En la primera se 
realiza una breve revisión de la literatura existente respecto del ascenso laboral femenino, su importancia y la manera en la que se ha frenado. En la segunda se lleva a cabo la cuantificación de la desigualdad numérica existente junto con una propuesta de lo que sucedería si una cuota de género fuese impuesta. Por último, se presentan las conclusiones.

\section{BREVE REVISIÓN DE LA LITERATURA}

En esta sección se realiza una breve revisión de la literatura que aborda teórica y empíricamente el ascenso laboral femenino. Esta revisión permitirá enfocar con claridad la justificación que lleva a contabilizar las posiciones en consejos de administración dado que el ascenso laboral es una herramienta para poder acceder a esos niveles jerárquicos dentro de las organizaciones. Al tocar este tema se busca establecer la relación entre la discriminación existente y la poca participación femenina en lo altos niveles de las empresas.

La literatura que aborda el ascenso laboral femenino se puede dividir en dos explicaciones teóricas. En primer lugar se encuentra la visión que asocia el ascenso laboral a su productividad y, en segundo, las que se enfocan en la discriminación por factores estructurales como gustos personales o prejuicios (Blau y De Varo, 2006: 4).

La visión que se relaciona con la productividad sugiere que los resultados del empleo como el salario y el propio ascenso laboral pueden no ser favorables para las mujeres debido a 
las diferencias en productividad respecto de los varones. En este sentido, la educación y la experiencia laboral previas juegan un papel importante para explicar el ascenso. Un problema que surge para probar este punto de vista es que la estadística asociada suele no estar disponible.

Por su parte, la explicación asociada a la discriminación por preferencias personales toma forma de prejuicio e implica que una autoridad prefiere trabajar con hombres en lugar de mujeres y por ello no les es otorgado un ascenso. En un entorno de discriminación, si la figura de autoridad recae en un hombre o un grupo de ellos, los ascensos laborales de las mujeres se pueden tornar aún menos probables. Esta situación se comprueba en el estudio de Bell, Smith y Vener (2008), quienes encuentran que el ascenso laboral de las mujeres es más probable en empresas que son lideradas por mujeres que en aquellas lideradas por hombres. Es decir, en empresas administradas por hombres la probabilidad de asignar un ascenso a un hombre es mayor y en el caso de las mujeres es menor.

Un elemento extra a considerar es el ni-

${ }^{3}$ Es por esto que existen estudios en los que el ascenso laboral femenino es enfocado desde la perspectiva de que otra mujer ocupe un alto cargo. Según Beckman y Phillips (2005) las mujeres son incluidas como socias en despachos jurídicos en los que laboraban como em pleadas un año después de que dicho despacho fuera contratado por una empresa en la que la dirección general o algún otro puesto de ese tipo estuviera ocupado por una mujer. vel, en el que se puede presentar la discriminación en el ascenso dentro de la jerarquía de la empresa La evidencia empírica de esta situación se puede hallar en Blau y De Varo (2006: 5), quienes afirman que la discriminación hacia mujeres se encuentra más acen- 
tuada en los niveles más altos al interior de las empresas. ${ }^{3}$

En general, la literatura que versa sobre la discriminación del ascenso laboral femenino es cuantiosa y la evidencia existe para diversos países y sectores. Collier (1994), señala que en los países en desarrollo la discriminación laboral contra las mujeres se manifiesta en forma de acceso diferenciado al empleo remunerado, a diferencia de los países desarrollados donde la discriminación toma forma de diferenciales en las tasas salariales. Por su parte, Okpara (2006) reporta que existen diferencias de género en la promoción laboral en la industria bancaria en Nigeria. El autor menciona que las mujeres reportan menor satisfacción que los hombres con las políticas de promoción que tienen los bancos de ese país.

La evidencia para países europeos se puede encontrar en Muehlau (2011), quien indica que la calidad de los empleos para varones se caracteriza por altos requerimientos técnicos, buenas oportunidades de ascensos y autonomía; mientras que para las mujeres domina una situación contraria. En este mismo sentido Pekkarinen y Vartiainen (2004) encontraron que las promociones son menores para las mujeres en la industria metálica y que éstas se hacen cargo de empleos de menor rango.

La evidencia para otros países como Canadá indica que las mujeres trabajadoras presentan 3\% menos posibilidad de ser promovidas que los hombres, según Javdani y McGee (2015). En el caso de los Estados Unidos Blau y De Varo (2006) anali- 
zan los ascensos de hombres y mujeres en ambientes urbanos. La base de datos se encuentra dividida por puesto, nivel educativo y otras variables. Y sus hallazgos apuntan a que las mujeres tienen menores probabilidades tanto en las expectativas de ascenso como en el ascenso mismo. La distinción entre la expectativa de ascender y el ascenso mismo resulta importante debido a que si la expectativa no es buena puede desincentivar a las mujeres a que lleven a cabo acciones para obtenerla.

En un estudio reciente en Finlandia, Cassidy, De Varo y Kauhanen (2016) utilizaron la teoría de señalización y le incorporan el aspecto del género para llegar a la conclusión de que las probabilidades de ascenso son más sensibles para mujeres con mayor nivel educativo pero aún así, son menores para las mujeres que para los hombres. Dada su importancia conviene profundizar un poco en la teoría de la señalización. Desde esta perspectova la educación puede identificarse con la información de la productividad que tendrían los empresarios de sus trabajadores, es decir, es un instrumento informativo para los empresarios.

Según Montoya y Montoya (2007: 11), la contratación es un proceso de inversión bajo incertidumbre en el cual contratar un empleado consiste en la compra de una lotería. Ello implica que en condiciones de neutralidad del riesgo el salario es tomado como la contribución marginal del individuo a la organización que lo contrata. Por su parte Addison et al. 
(2014), considera que las mujeres que tienen menores niveles de educación poseen pocas o nulas posibilidades de ascender comparadas con las que poseen mayores niveles educativos. Estos resultados coinciden con los de Hashimoto y Sato (2014), quienes encuentran una notable diferencia entre el poco ascenso laboral de mujeres con educación superior respecto de las que no tienen grados académicos.

En la literatura especializada se habla de empleo de alto nivel que engloba empleo ejecutivo, gerencial y membresía a consejos de administración. Stier y Yaish (2014) exploran las diferencias de género en la calidad del empleo de las mujeres ejecutivas incluyendo sus ascensos. Los autores encuentran un rezago importante en favor del género masculino. Además, el estudio halla que las mujeres son compensadas con la provisión de mejores condiciones laborales en su actual posición en la empresa. Ma (2004) encontró que las mujeres estuvieron poco representadas en los más altos rangos y sobrerrepresentadas en los rangos menores en empresas financieras en Inglaterra. La autora también concluyó que los salarios pagados a las mujeres no son tan altos como los de los hombres excepto en los empleos de mayor rango.

Si además se incluye la variable tamaño de empresa, la discriminación laboral en contra de las mujeres se acentúa. Existe evidencia que afirma que las políticas de segregación laboral en las que son favorecidos los hombres son más visibles en las organizaciones de mayor tamaño (Baron y Bielby, 1985: 247). 
En este punto conviene ofrecer un panorama de la situación de las mujeres empresarias y ejecutivas en México. Al respecto la literatura reporta trabajos como los de Zabludovsky (2013), que realiza una amplia revisión de diversos temas como son las metodologías empleadas en algunos estudios en los que se aborda la cultura empresarial y el liderazgo femenino. Resalta la escasa participación de las mujeres en las cámaras empresariales. Por su parte sobresalen las diferencias regionales, pues en entidades como Yucatán y Jalisco la participación femenina destaca por ser mayor. Siguiendo con la óptica regional, se puede citar el estudio de Arias (2007) en el que se compara la esfera profesional rural en ciudades de México y Argentina. El estudio concluyó que esa tipología de mujeres tiene la seguridad de que en su actividad económica se encontrarán diferencias y jerarquías con el género masculino.

Para el caso de las mujeres ejecutivas, aunque no necesariamente miembros del consejo de administración, Pérez y García (2006: 8) encuentran que solo un $10 \%$ de ellas tienen el nivel ejecutivo en empresas internacionales que operan en México. Las autoras remarcan el hecho de que las mujeres tienen dificultades de acceso a puestos directivos en corporaciones de capital extranjero. En el caso de ciertos sectores como el bancario, Martínez (2007) afirma que la primera mujer que accedió a un puesto de alta gerencia en dicho sector se dio después de la nacionalización de la banca en 1982. Un estudio de particular relevancia es el llevó a cabo Hernández (2010: 57), en 
el que se resaltan nuevas tendencias en el papel de las mujeres en el ambiente empresarial. El estudio mencionado indica que existe una relación negativa entre número de mujeres con actividad empresarial y tamaño de empresa, pues la mayoría de las mujeres empresarias tienen microempresas y muy pocas aparecen en grandes empresas.

Se considera necesario mencionar que la información es muy escasa para el caso específico de México. De las pocas referencias está la encuesta de la Consultora de Recursos Humanos ADECCO (2013), que indica que $22 \%$ de las mujeres encuestadas no se sentía exitosa en su ámbito laboral. Entre las razones de las formas se encuentra la preferencia que existe en favor del género masculino en ciertas posiciones. La encuesta no menciona qué puestos son, pero confirma la preferencia del sexo masculino para algunas posiciones laborales.

Resulta conveniente enlistar los elementos que se han revisado y que servirán para establecer la hipótesis a probar. Primero, la discriminación en contra de las mujeres existe en una gran cantidad de sectores y ámbitos de la vida nacional; segundo, la discriminación en el ascenso laboral femenino es una práctica común en muchas partes del mundo y en diversos sectores económicos; tercero, la preferencia por ascender hombres se acentúa tanto en las esferas más altas de la estructura administrativa de las empresas como en las empresas de mayores dimensiones; cuarto, en empresas administradas por hombres las probabilidad de ascensos para mujeres son menores; y quinto, 
en México, 22\% de las mujeres reportan no sentir éxito laboral, pues mencionan preferencia por el sexo masculino en el acceso a ciertos puestos laborales.

Con los elementos mencionados la hipótesis que se pretende probar es: la conformación de los consejos de administración en grandes empresas en México se caracteriza por tener una marcada inequidad de género en favor del sexo masculino.

Antes de continuar se considera pertinente hablar sobre los costos que pude tener no incluir mujeres en empleos de alto nivel. Este costo puede traducirse en una ralentización en el desempeño general de la empresa, pues el empleo femenino de alto nivel parece estar asociado positivamente con un mejor desempeño de las empresas. A dicho resultado llegan los estudios de Krishnan y Park (2005), Smith y Verner (2006), Dezsö y Gaddis (2008), Abramovitz (2012). Los autores coinciden en mencionar que el impacto de las mujeres en los empleos de alto nivel está condicionado sensiblemente por sus habilidades y estudios.

La economía en general también puede resultar afectada por esta situación toda vez que autores como Klasen (1999) mencionan que el sesgo de género en el empleo está asociado a bajos niveles de crecimiento económico en el sur de Asia y África subsahariana. El cálculo de los autores indica que el crecimiento de la economía de la región se pudo haber reducido en 3\% durante el periodo que va de 1960 a 1992. En este mismo sentido, el Fondo Monetario Internacional (FMI 2013: 
5) afirma que la existencia de "mejores oportunidades para que las mujeres obtengan más ingresos contribuye a un desarrollo económico más amplio en las economías en desarroIlo". Por otro lado Esteve (2004) halla evidencia de que la discriminación de género en empleos a nivel gerencial puede frenar la producción por habitante. Según la autora, la generalidad es que la discriminación laboral, desde el acceso a puestos de baja responsabilidad hasta el ascenso a posiciones gerenciales, tiene efectos económicos negativos como frenar la producción por habitante de una nación. La investigadora concluyó que ese efecto puede no ser pequeño.

Por otra parte, se considera importante analizar algunos rasgos del objeto de estudio que es el consejo de administración. Un consejo de administración es un órgano de gobierno que poseen las empresas en todo el mundo. Esta estructura administrativa sirve para velar por los intereses de los accionistas haciendo uso de la alta dirección. Entre las funciones que puede tener un consejo de administración están: evaluar metas establecidas, toma de decisión respecto de alianzas estratégicas, inspeccionar altos directivos, entre otras.

En México, la Ley del Mercado de Valores (LMV) se encarga de regular a los consejos de administración. En dicha ley se reconocen al menos tres tipos de consejeros: propietarios, suplentes

${ }^{4}$ Según el Instituto Mexicano de Mejores Prácticas Corporativas (IMMPC), Los consejeros propietarios pueden ser aquellos que representan a los accionistas pero que no poseen acciones; el suplente adquiere funciones cuando el propietario no puede asistir a las sesiones del consejo y el consejero independiente no tiene una relae independientes. ${ }^{4}$ La figura de conse- 
jero independiente fue introducida en 2005 a la LMV. De acuerdo con dicha ley, la máxima cantidad de consejeros posibles permitidos es veintiuno, de los cuales al menos el veinticinco por ciento deben ser independientes. De acuerdo con Silva y Alonso (2013: 289) "la tarea clave de un consejero independiente es evitar que los accionistas de control incurran en abusos en detrimento de los minoritarios". El artículo 26 de la misma ley indica que el consejero independiente debe ser seleccionado por su experiencia, capacidad y prestigio profesional. Como se puede apreciar, ser nombrado(a) miembro de un consejo de administración, al menos como consejero independiente, requiere ser una persona que tenga cierta cantidad de tiempo dedicada a actividades en las que haya sobresalido de manera notable y poseer una experiencia tal que sus resultados le puedan dar el prestigio que requiere la ley. Se considera que la experiencia mencionada no se puede obtener de otro modo que no sea con el trabajo en una o diversas áreas de una o varias empresas.

Cabe mencionar que, de acuerdo a la LMv, los consejeros independientes deben estar libres de conflicto de intereses y no deben estar supeditados a intereses per${ }^{5}$ Esto significa que el consejero no debe ser ni cliente, ni proveedor, ni empleado. sonales, patrimoniales ni económicos. ${ }^{5}$ Además, no deben tener lazos de consanguinidad con ningún otro miembro del consejo de administración. Es decir, formar parte de un consejo de administración bajo la figura de consejero indepen- 
diente no puede llevarse a cabo sino a través de ascensos laborales. A diferencia, los consejeros propietarios pueden pertenecer a un consejo dada su relación de consanguinidad.

\section{CUANTIFICACIÓN DE LA} DESIGUALDAD NUMÉRICA EN LA COMPOSICIÓN DE CONSEJOS DE ADMINISTRACIÓN

Para conocer el grado de igualdad numérica de género en puestos de consejos de administración de empresas que operan en México se consultaron y contabilizaron los consejos de administración de empresas que cotizan en la Bolsa Mexicana de Valores (BMV). Se han tomado en cuenta estas empresas por varias razones. En primer lugar, porque la información de los miembros de sus consejos es de fácil acceso. En segundo, se reportó evidencia en párrafos anteriores que en este tipo de empresas la discriminación hacia las mujeres es mayor que en empresas de menores tamaños. A este respecto se puede decir que las micro o pequeñas firmas pueden no tener un consejo de administración, ${ }^{6}$ y en caso de tenerlo, la información de los nombres de sus miembros y su tipo es de difícil acceso, pues no están obligadas a hacer públicos los nombres de sus integrantes. Cabe hacer notar que todas las empresas que cotizan en bolsa tienen la característica de ser empresas de gran tamaño. 
Se ha dividido el análisis por tipo de consejero en tres rubros: consejero-propietario, consejero-suplente y consejero-independiente. Cuando la información no ha permitido hacer la distinción de los rubros mencionados se ha optado por no tomarla en cuenta debido a que se puede incurrir en un sesgo que llevaría a sobre o subestimar el resultado del conteo. ${ }^{7}$

Se sumó el total de consejeros reportados por rubro. De dicho total se obtuvo el porcentaje de mujeres. Para el caso de las consejeras propietarias resultó que solamente $6.7 \%$ fueron mujeres, el porcentaje de consejeras suplentes fue de $8 \%$ y en el rubro de consejeras independientes fue de 3.8 por ciento. Estos resultados indican que el grado de desigualdad numérica de género en la composición de los consejos de administración es muy grande. Los porcentajes de cada rubro se muestran en la Tabla 1.

Otros datos derivados del análisis de la base de datos son que $70 \%$ del total de consejos de administración tienen cero mujeres en el rubro de consejeras propietarias mientras que $20 \%$ tienen una mujer propietaria y $10 \%$ tienen dos o más mujeres propietarias. Por su parte, en el rubro de miembros suplentes, el porcentaje de empresas que tienen cero mujeres consejeras es $62 \%$, las que tienen una mujer suplente $25 \%$ y $13 \%$ tienen dos o más consejeras suplentes. En el rubro de independientes $80 \%$ reportaron cero mujeres miembros, $15 \%$ tuvieron una consejera y $5 \%$ dos mujeres o más. Cabe mencionar que el número promedio de lugares de los consejos de 
Tabla I. Distribución de mujere en consejos de administración

\begin{tabular}{|l|l|l|l|}
\hline & \multicolumn{3}{|l|}{ Tipo de consejeras } \\
\cline { 2 - 4 } & Propietarias & Suplentes & Independientes \\
$\begin{array}{l}\text { Porcentaje de mujeres miembros de con- } \\
\text { sejo }\end{array}$ & 67 & 8 & 3.8 \\
Porcentaje de consejos con cero mujeres & 70 & 62 & 80 \\
Porcentaje de consejos con una mujer & 20 & 25 & 15 \\
Porcentaje consejos con dos o más mu- & 10 & 13 & 5 \\
\hline jeres
\end{tabular}

Fuente: elaboración propia con datos de las empresas.

administración en el rubro de propietarios es de 6.76 miembros, 6.15 en el rubro de suplentes y de 5.62 en independientes.

El pequeño porcentaje de mujeres miembros de un consejo de administración hallado para los tres rubros ha cambiado muy poco en los últimos años. En un estudio previo Santos y Castañón (2003) reportan la base de datos de los miembros de consejos de administración de empresas que cotizan en la BMv, de donde se desprende que solamente el $4.5 \%$ son mujeres. El estudio no hace distinción sobre el tipo de consejeros ni menciona cuáles son las empresas que tienen mayor o menor cantidad de mujeres como miembros de sus consejos ad- 
ministrativos. Se considera que dicho porcentaje es muy parecido a los mostrados en la Tabla 1.

Para dimensionar los porcentajes se citará el estudio del IESE Business School (2016) Ilamado Informe de las mujeres en los consejos del IBEX-35. Dicho estudio reporta el número de mujeres en consejos de administración en empresas españolas que cotizan en el IBEX-35. Sus resultados arrojan que en 2016 hubo un total de $19.83 \%$ de mujeres que ocuparon un lugar en dichos consejos. El mismo porcentaje, pero en el 2010 fue de $10.56 \%$. Claramente son porcentajes superiores a los encontrados en México.

En el mismo estudio se menciona que en el resto de la Unión Europea el peso de las mujeres en los consejos de administración es mayor, pues en 2015 alcanzó un promedio de $21.2 \%$ mientras que en 2010 fue de $11.9 \%$. Particularmente Francia alcanzó un 33\% de mujeres aunque ninguno de los porcentajes mencionados distingue tipos de consejeras. Otro dato que puede ayudar es el reportado por Bell (2005: 5), quien encuentra que el porcentaje de mujeres en los cinco puestos más altos de empresas norteamericanas en el periodo 19922003 ascendió a $4.45 \%$. Aunque la autora no menciona si tales ejecutivas son parte del consejo de administración, sí se puede tomar el dato como comparable dado que se habla de los niveles más altos en puestos de administración de empresas privadas. Con la misma fuente de datos, pero para el periodo 1992-1997, Bertrand y Hallock (2001) reportan que solo 


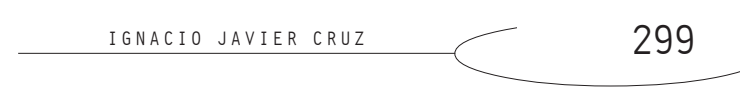

$2.5 \%$ de mujeres ocuparon un puesto dentro de los cinco ejecutivos de mayor rango en las empresas inspeccionadas indicando un crecimiento en el número de mujeres que ocuparon dichos puestos.

Para el caso de los Estados Unidos, el reporte de Catalyst (2016) indica que $19.9 \%$ de mujeres ocupan un asiento en algún consejo de administración de las empresas que conforman el índice Standar and Poor's 500, siendo estos resultados muy parecidos a los hallados en el IBEX-35. ${ }^{8}$

${ }^{8}$ Los resultados mostrados para España, Estados Unidos y México se consideran comparables, pues se habla de empresas que cotizan en algún índice bursátil.

Es necesario mencionar que la presencia de mujeres en algunos consejos de administración puede no deberse al ascenso laboral sino a que la empresa forma parte de su patrimonio. A este respecto, un dato que no debe dejarse de lado es que, en el rubro de consejeros propietarios $31.5 \%$ de los consejos de administración que tienen una o más mujeres, sus apellidos coinciden con el del presidente del consejo. El caso de Interceramic es una muestra de esta situación, pues hay cuatro consejeras propietarias cuyo apeIlido paterno coincide con el del presidente del consejo de administración. Además están reportadas como consejeras patrimoniales, ${ }^{9}$ lo que sería evidencia clara de su re${ }^{9}$ Un consejero patrimonial es aquel que además de ser miembro del consejo de administración posee lación de consanguinidad.

Se realizó por separado un conteo en el que se dejó de lado a las mujeres que presentaron el mismo apellido que el presi- 
dente del consejo con el objetivo de observar el porcentaje de mujeres que pudieron llegar al consejo sin una relación de consanguinidad. El resultado para el rubro de consejeras propietarias fue de $5.7 \%$ mientras que el mismo ejercicio para consejeras suplentes fue de $6.4 \%$ y cero por ciento en el rubro de consejeras independientes. Es decir, en aquellas situaciones en donde no hay razón de consanguinidad para incluir mujeres, éstas se encuentran con menor frecuencia.

De los consejos estudiados destacan Bachoco, CiBanco, Corpovael, Corporativo GBM, Finamex, Grupo Industrial MASECA, Grupo famsA, Grupo Comercial Gomo, Grupo Posadas, Grupo Financiero Multiva, Grupo Profuturo, Grupo Hotelero Santa Fe, Grupo Bмv, Hilasal, Infraestructura Energética Nova, Proteak Uno, RHL Propierties, Santander, Invex y Banregio y Value Grupo Financiero. En ninguno de estos casos se reportan ni una sola mujer miembro del consejo ni como propietaria, suplente o independiente. En el rubro de propietarios destaca Interceramic que presenta un $50 \%$ de mujeres en su consejo. Esta es la empresa con el mayor porcentaje de consejeras, Grupo GAMESA 27\%, Corporativo Fragua 28\%, Grupo Radio Centro 28\%. Con la salvedad de que el total de mujeres consejeras de las empresas mencionadas tiene relación consanguínea con el presidente del consejo. En este sentido resalta kof que presenta $40 \%$ consejeras sin que sus apellidos denoten relación de consanguinidad. 
En el rubro de suplentes, Wal-Mart destaca por presentar $25 \%$ de consejeras y Axtel $29 \%$. En el rubro de independientes destacan Gas Natural FENOSA, que reporta un $43 \%$ de consejeras, Grupo Lala 25\%, y Compartamos con $29 \%$.

La posibilidad de romper con la desigualdad numérica de género en la conformación de consejos de administración, al menos de las empresas de gran tamaño, se podría encontrar en la introducción de una norma o ley que estableciera una cuota de género en el número de mujeres que formasen parte de un consejo de administración sin modificar los requisitos de experiencia y prestigio que menciona actualmente la LMV. Según Smith, (et al., 2012: 2) en Noruega existe una norma que indica que al menos $40 \%$ de los miembros de la junta directiva deben ser mujeres. Según los mismos autores, la discusión sobre la composición de dicha junta se ha dado intensamente en otros países como Suecia y Dinamarca.

Con el objetivo de cuantificar el porcentaje de mujeres que llegarían a formar parte de un consejo de administración, se realizaron ejercicios simples de simulación que buscaron calcular el promedio de mujeres en consejos solamente para el rubro de independientes en los que se supusieron diversas cuotas de género. La explicación del por qué se llevó a cabo este análisis solamente para consejeros independientes es que en la LMV se mencionan los requisitos para lograr un lugar en el consejo, que fueron previamente identificados con ascenso laboral. En cambio, no existen requisitos para ser nombra- 
do consejero propietario o suplente y no es el objetivo de esta investigación tratar de delinearlos. De esta situación se desprende que incentivar la inclusión de consejeras independientes sería una acción que estimularía el ascenso laboral femenino en la parte más alta de las estructuras administrativas de las empresas en México.

En la primera cuota de género se supuso que la LMV obliga a que, como mínimo, una mujer ocupe un lugar como consejera independiente. En este caso existen dos posibilidades: la primera es que las empresas mantengan el número de consejeros que reportan y, por tanto, un hombre debería ser sustituido por una mujer; y la segunda posibilidad es que las empresas incrementen el número de consejeros incorporando un lugar destinado a una mujer. Para ambos casos no se modificaron los consejos de las empresas que presentaron al menos una

\begin{tabular}{|l|l|l|}
\cline { 2 - 3 } \multicolumn{1}{c|}{} & \multicolumn{2}{|l|}{$\begin{array}{l}\text { Tabla 2. Modificación de porcentajes en } \\
\text { consejos de administración }\end{array}$} \\
\cline { 2 - 3 } & $\begin{array}{l}\text { Al menos una mujer } \\
\text { miembro }\end{array}$ & $\begin{array}{l}\text { Al menos una tercera } \\
\text { parte }\end{array}$ \\
\hline Hombre sustituido por mujer & 25.14 & 24.65 \\
\hline $\begin{array}{l}\text { Incremento de un lugar en } \\
\text { consejo }\end{array}$ & 19.8 & 16.18 \\
\hline
\end{tabular}

Fuente: Elaboración propia con datos de las empresas. 
mujer consejera independiente pues no tendrían incentivo para hacerlo. Los resultados se pueden observar en la Tabla 2. mujeres consejeras independientes pasaría de 3.8 a $25.14 \%$, mientras que para la segunda posibilidad el porcentaje sube a 19.8\%. En ambos casos el porcentaje obtenido es similar o superior al de países como España y Estados Unidos.

En el segundo ejercicio el cálculo se realizó suponiendo que la ley obliga a que al menos una tercera parte de los consejeros independientes deban ser mujeres. Los cálculos arrojan que, en el caso para el cual las empresas reaccionan sustituyendo hombres con mujeres, el porcentaje sería de $24.66 \%$, mientras que si se abren nuevos lugares ocupándolos mujeres el porcentaje sería de $16.18 \%$. De este ejercicio se desprende que solamente con una sustitución de consejeros el porcentaje supera al reportado por países como España y los Estados Unidos.

Como se pudo observar, una modificación a la ley del tipo descrito en este apartado podría traer beneficios para las mujeres, las empresas y hasta el crecimiento económico del país. Queda en adelante, por un lado, encontrar más literatura tanto teórica como evidencia empírica para apuntalar la revisión de literatura. Por otro lado sería pertinente incluir en el análisis otras empresas grandes que no coticen bolsa de valores para corroborar los resultados encontrados, así como cuantificar la composición en los consejos de administración en empresas de menor tamaño. 


\section{CONCLUSIONES}

El objetivo de este artículo fue mostrar la desigualdad numérica en la composición de los consejos de administración de grandes empresas en México. Se consideró que formar parte de un consejo de administración como consejero independiente implica que una persona ha pasado por un proceso de ascensos laborales en su carrera. Particularmente, el freno al ascenso laboral es una realidad que la literatura ha reportado con claridad en países y sectores alrededor del mundo manifestándose una clara discriminación en favor del sexo masculino. Además, la discriminación se ve acentuada cuando existen dos variables extras: el tamaño de la empresa y que la preferencia por el sexo masculino se da con mayor frecuencia en los círculos más altos de la estructura administrativa.

Aunque se debe tener cuidado con la interpretación porque la desigualdad numérica de los consejos no es lo mismo que la discriminación en los ascensos laborales. Se consideró que lo segundo en una herramienta para conseguir lo primero.

En el caso de las empresas que cotizan en la Bolsa Mexicana de Valores, los consejos mostraron estar compuestos por personas del sexo masculino en una abrumadora mayoría. La desigualdad numérica mostrada se dividió en tres rubros: consejeras propietarias, suplentes e independientes. La diferenciación se debió a que así lo reporta la información y a que la 
condición de cada tipo de consejero es diferente. El porcentaje de mujeres en el total de miembros propietarios es $6.7 \%$, el de suplentes es $8 \%$ y el de independientes $3.8 \%$ tales datos demuestran la enorme desigualdad numérica en la conformación de los consejos en favor del género masculino.

Una política que se puede llevar a cabo con el objetivo de disminuir o erradicar la desigualdad encontrada sería introducir una ley o norma que impusiera una cuota de género en los consejos de administración. La simulación llevada a cabo encontró que de existir alguna cuota de género, los porcentajes de mujeres ocupantes de un lugar en consejo de administración tendrían un nivel similar a los que poseen España o Estados Unidos. De esta manera disminuiría considerablemente la desigualdad de género y fortalecería al ascenso laboral como herramienta de superación de las mujeres.

Bibliografía

Abramovitz, A. (2012). Are women impact players?. The effect of female executive on firm performance and capital structure. Senior thesis. Claremont McKenna College.

AdECCO. (2013). Mujer mexicana, éxito y competitividad laboral.

ARIAS, P. (2007). "Mujeres en los negocios y mujeres de negocios". En Barrera, Dalia "Empresarias y ejecutivas, mujeres en el poder». El Colegio de México, México 
BARON, J. y BIELBY, W. (1985). Organizational barriers to gender equality: sex segregation of Jobs and Opportunities en Gender and the life course. (Alice Rossi, 1985). American Sociological Association.

BeCKMAN, Ch. y PHILLIPS, D. (2005). Interorganizational Determinants of promotion: Client Leadership and the Attainment of Women Attorneys. American Sociological Review, vol. 70, No. 4, pp. 678-701.

BELL, L. (2005). Women led firms and the gender gap in top executive jobs. Discussion Paper Series IZA DP No. 1689.

SMith, N. y Verner, M. (2008). Gender Differences in promotion into top-management jobs, Working Papers No. 08-21, Universidad de Aarhus.

Bertrand, M. y Hallock, K. (2001). The Gender in top corporate jobs. Human Resource Studies. Cornel University.

BLAU, F. y DE VARO J. (2006). New evidence on gender differences in promotion rates: An empirical analysis of a simple of new hires. Working paper No. 891, Princeton University.

CASSIDY, H.; De Varo, J. y Kauhanen, A. (2016). Promotion signaling, gender, and turnover: New Theory and evidence. Journal of Economic Behavior and Organization, Vol. 126, pp. 140-166.

CATAlYST (2016). Pyramid: Women in S\&P 500 companies. New York: Catalyst, September 19.

ColLIER, P. (1994). "Gender aspects of Labor allocation during structural adjustment: Theoretical Framework and the Africa experience". In Labor markets in an era of adjustment, vol. 1, ed. By Susan Horton, R. Hanbur and D. Mazumbur. Washington. 
Dezsö, C. y GadDIS, D. (2008). Girl power: Female Participation in Top Managment and Firm performance. Working paper.

EsteVE, B. (2004). Gender Discrimination and Growth: Theory and evidence from India, Working Paper DEDPS 42.

FONDOMONETARIO INTERNACIONAL. (2013). Las mujeres, el trabajo y la economía: beneficios macroeconómicos de la equidad de género. Documento de análisis del personal técnico del FMI.

HASHIMOTO, Y. у SATO, K. (2014). Wages, promotions and gender workplace Segregation. Economic Review, vol. 65, núm. 3, pp. 221-237.

HeRnández, E. de la P. (2010). Nuevas Tendencias en el mundo empresarial: La participación de las mujeres. Revista de Estudios de Género. La ventana, vol. Iv, núm. 32, pp. 52-80.

IESE BUSINESS SCHOOL. (2016). Informe de las mujeres en los consejos de administración del IBEX-35.

JaVDANI, M. y McGeE, A. (2015). Moving up or Falling behind? Gender, Promotions, and Wages in Canada. IZA Discussion Paper No. 9380. KLASEN, S. (1999). Does Gender inequality reduce growth and development? Evidence from cross-country regressions. Working Papers Series No.7, Policy Research Report on Gender and Development. The World Bank. KPMG. (2013). Empresas Familiares en México. El desafío de crecer, madurar y pertenecer.

KRISHNAN, H. y PARK, Daewoo. (2005). A few good women on top management teams. Journal of Business Research, vol. 58, núm. 12, pp. 17121720.

MA, ADA. (2004). Gender discrimination pay and promotion in job ladders. Royal economic, Society Annual Conference, No. 157. 
Martínez, G. (2007). “Ejecutivas: una nueva presencia en los espacios de poder". En Barrera, Dalia. Empresarias y ejecutivas, mujeres en el poder. El Colegio de México, México.

MontoyA, I. y Montoya, L. (2007). Reflexiones para una elaboración conceptual del proceso de chantaje, a partir del problema de la selección adversa. Ecos de Economía, No. 24. Abril, pp. 8-26.

Muehlau, P. (2011). Gender inequality and job quality in Europe. Management Revue Socio-Economic Studies, vol. 22, núm. 2, pp. 114-131.

Navarro, D.; Narro, J. y Orozco, L. (2014). La mujer en México: Inequidad, pobreza y violencia, Revista Mexicana de Ciencias Políticas y Sociales, Vol. 59, No. 220, pp. 117-146.

OKPARA, J. (2006). Gender and the relationship between perceived fairness in pay, promotion and job satisfaction in Sub-Sahara African economy. Women in Management Review, Vol. 21, núm. 3, pp. 224-240.

PeKkARINen, T. y VARTIAINEN, J. (2004). Gender differences in job assignment and promotion in a complexity ladder of Jobs, Discussion Paper Series No. 1184. IZA Bonn.

Pérez, M. y García, J.L. (2006). La formación de los ejecutivos de las grandes corporaciones y los procesos de globalización. Iberoforum. Revista de Ciencias Sociales de la Universidad Iberoamericana. Vol. 1, núm, II, pp. 1-18.

SANTOS, I. y CASTAÑón, G. (2003). La élite del poder económico en México. Estudio de la red de consejeros de administración de las empresas que cotizan en bolsa. Perspectivas. Revista de análisis en economía, comercio y negocios internacionales. 
SILVA, J. y ALonso, E. (2013). Las funciones de los consejeros independientes de las sociedades en México: recomendaciones para el cambio a partir de las asimetrías existentes entre la práctica y la ley. Boletín Mexicano de Derecho Comparado, vol. 45, no. 136, pp. 287-316.

SMith, N.; SMITH, V. y Verner, M. (2006). Do women in top managements affect firm performance? A panel study of 2,500 Danish firms. International Journal of Productivity and Performance Management, vol. 55, núm. 7, pp. 569-593.

- (2012). Women in top Management and Firm performance. Working Paper 08-12. Departamento de Economía, Universidad de Aarhus.

STIER, H. y YAISH. M. (2014). Occupational segregation and gender inequality in job quality: a multi-level approach. Work, employment and society, vol. 28, núm 2, pp. 225-246.

VILLALOBOS, S. (2016). Igualdad y trabajo pastoral: La experiencia de las mujeres en los ministerios religiosos ordenados y consagrados en México. Revista Interdisciplinaria de Estudios de Género, Año 2, núm. 4, pp. 76-102.

Zabludovsky, G. (2013). Empresarias y ejecutivas en México. Diagnósticos y desafíos, Plaza y Valdés, México.

\section{Anexo}

A continuación se enlistan los consejos de administración consultados que sumaron un total de 137. ${ }^{10}$

Accel, Actinver, Aeroméxico, Agro Industrial Exportadora, Altos Hornos de México, Alsea, Arca Continental, Alfa, Alpek, América Móvil, Axtel, 
Autlán, Bachoco, Banorte, Banregio, Bimbo, Bio Pappel, Cablevisión, Cemex, CiBanco, CMr, Controladora Comercial Mexicana, Consorcio Aristos, Corpovael, Corporación Interamericana de Entretenimiento, Corporativo Fragua, Corporativo GMB, Corporación GEO, Crédito Real, Consocio ARA, CYDSA, Desarrolladora Homex, DINE, Edoardos Martín, Elementia, Farmacias Benavides, FEMSA, FINAMEX, Financiera Independencia, Grupo BMv, GNP, Gas Natural fenosA, Fresnillo PLC, Genomma Lab, General de Seguros, Grupo Comercial Gomo, Grupo Modelo, Gruma, Grupe, Grupo Carso, Grupo Industrial MASECA, Grupo Famsa, Grupo Financiero Bancomer, Grupo Financiero Banamex, Grupo Financiero Inbursa, Grupo Financiero Multiva, Grupo Senda, Grupo financiero Interacciones, GNP, Grupo Sanborns, Grupo Posadas, Grupo Cementos Chihuahua, Grupo Elektra, Grupo Gamesa, Grupo Comercial Chedrahui, Grupo Lala, Grupo Sports World, Grupo Rotoplas, Grupo Radio Centro, Grupo Aeroportuario del Pacífico, Grupo Aeroportuario del Sureste, Grupo Bafar, Grupo Mexicano de Desarrollo, Grupo Qumma, Grupo Diniz, Grupo Herdez, Grupo Gigante, Grupo Profuturo, Grupo México, Grupo BMv, Grupo Vasconia, Grupo Industrial Saltillo, Grupo Hotelero Santa Fe, Grupo Kuo, Grupo Pocheteca, Hilasal, Hoteles City Express, HSBC, Industrias Peñoles, Industria Automotriz, ICA, IDEAL, Interceramic, Invex, Javer Casas, Kimberly Clark de México, KOF, Liverpool, Maxcom, Megacable, Mexichem, Minera Frisco, Nemak, Promotora Ambiental, Promotora y Operadora Infra, Peña Verde, Planigrupo latAM, OHL México, Organización Cultiba, Organización Soriana, QB industrias, Qualitas Controladora, Rossini, RHL Propierties, Proteak Uno, Santander, Sare Holding, Sigma Ali- 
mentos, Telmex, Televisa, Tenaris, Teakcheam, TV Azteca, Transportación Marítima Mexicana, Telesites, Unifin, Urbi, Value Grupo Financiero, Ve Por Más, Vesta Inmobiliaria, Volaris, Vitro y Walmart México y Centroamérica, 